\title{
N-Deacetylation of Sinorhizobium meliloti Nod Factors Increases Their Stability in the Medicago sativa Rhizosphere and Decreases Their Biological Activity
}

\author{
Christian Staehelin, ${ }^{1}$ Michael Schultze, ${ }^{1}$ Ken Tokuyasu, ${ }^{2}$ Véréna Poinsot, ${ }^{3}$ Jean-Claude Promé, ${ }^{3}$ \\ Éva Kondorosi, ${ }^{1}$ and Ádám Kondorosi ${ }^{1}$ \\ ${ }^{1}$ Institut des Sciences Végétales, Centre National de la Recherche Scientifique, Avenue de la Terrasse, \\ F-91198 Gif-sur-Yvette Cedex, France; ${ }^{2}$ National Food Research Institute, Tsukuba, Ibaraki 305, Japan; \\ ${ }^{3}$ Laboratoire des IMRCP, UMR 5623, Université Paul Sabatier, 118 Route de Narbonne, 31062 Toulouse \\ Cedex, France \\ Accepted 24 September 1999.
}

Nod factors excreted by rhizobia are signal molecules that consist of a chitin oligomer backbone linked with a fatty acid at the nonreducing end. Modifications of the Nod factor structures influence their stability in the rhizosphere and their biological activity. To test the function of $\mathrm{N}$-acetyl groups in Nod factors, NodSmIV $\left(\mathrm{C}_{16: 2}, \mathrm{~S}\right)$ from Sinorhizobium meliloti was enzymatically $\mathbf{N}$-deacetylated in vitro with purified chitin deacetylase from Colletotrichum lindemuthianum. A family of partially and completely deacetylated derivatives was produced and purified. The most abundant chemical structures identified by mass spectrometry were $\operatorname{GlcN}\left(\mathrm{C}_{16: 2}\right)$-GIcNAc-GlcNH${ }_{2}-\operatorname{GlcNAc}(\mathrm{OH})(\mathrm{S}), \operatorname{GlcN}\left(\mathrm{C}_{16: 2}\right)-$ GlcNAc-GlcNH $-\mathrm{GlcNH}_{2}(\mathrm{OH})(\mathrm{S})$, and $\operatorname{GlcN}\left(\mathrm{C}_{16: 2}\right)-$ $\mathrm{GlcNH}_{2}-\mathrm{GlcNH}_{2}-\mathrm{GlcNH}_{2}(\mathrm{OH})(\mathrm{S})$. In contrast to NodSmIV $\left(\mathrm{C}_{16: 2}, \mathrm{~S}\right)$, the purified $\mathrm{N}$-deacetylated derivatives were stable in the rhizosphere of Medicago sativa, indicating that the $\mathrm{N}$-acetyl groups make the carbohydrate moiety of Nod factors accessible for glycosyl hydrolases of the host plant. The $\mathbf{N}$-deacetylated derivatives displayed only a low level of activity in inducing root hair deformation. Furthermore, the N-deacetylated molecules were not able to stimulate Nod factor degradation by $M$. sativa roots, a response elicited by active Nod factors. These data show that $\mathrm{N}$-acetyl groups of Nod factors are required for biological activity.

Additional keyword: lipo-chitooligosaccharides.

Rhizobia interact with their leguminous host plants, e.g., Sinorhizobium meliloti establishes nitrogen-fixing symbiosis with Medicago sativa (alfalfa) (Bladergroen and Spaink 1998; Cohn et al. 1998; Schultze and Kondorosi 1998). Legume roots secrete (iso)flavonoids that induce the synthesis of spe-

Corresponding author: Christian Staehelin; Present address: Botanisches Institut, Universität Basel, Hebelstrasse 1, CH-4056 Basel, Switzerland; Telephone: +41-61-267 23 18; Fax: +41-61-267 23 30; E-mail: Chr.Staehelin@unibas.ch

Present address of Michael Schultze: The Plant Laboratory, Department of Biology, University of York, Heslington, York YO10 5YW, UK cific nodulation signals called Nod factors (NFs). They are modified lipo-chitooligosaccharides, i.e., $\beta$-1,4-linked oligomers of $N$-acetyl-D-glucosamine, with a fatty acid replacing the $N$-acetyl group on their nonreducing end (Long 1996; Promé 1996). Structural modifications of NFs at the reducing or nonreducing end have been shown to be important determinants of host specificity. For example, the sulfate group on the reducing end of NFs from $S$. meliloti is required for nodulation of Medicago sativa, while mutant strains producing nonsulfated NFs gain the ability to form nodules on Vicia sativa, a nonhost plant of $S$. meliloti (Roche et al. 1991). Based on the examination of plant responses to NFs, such as root hair deformation, it is generally thought that structural modifications of NFs influence their binding to putative plant receptors. Moreover, these modifications may affect the stability of NFs in the rhizosphere of the host plant and protect their carbohydrate moiety against degradation and inactivation by plant chitinases and other glycosyl hydrolases (Staehelin et al. 1994a, 1994b; Minic et al. 1998; Schultze et al. 1998). In $M$. sativa roots, NFs are hydrolyzed by a specific "lipodisaccharide forming" NF hydrolase. The activity of this glycosyl hydrolase is stimulated by NFs from $S$. meliloti at nanomolar concentrations, indicating that NFs induce their rapid degradation in the rhizosphere of the host plant (Staehelin et al. 1995, 1997).

The oligosaccharide core structure of rhizobial NFs is synthesized by NodC, an $\mathrm{N}$-acetylglucosaminyltransferase, that has sequence homologies to chitin synthases (Geremia et al. 1994; Kamst et al. 1999). This suggests that a core structure of $\mathrm{N}$-acetylglucosamine oligomers is essential for the biological activity of NFs. However, the requirement of the $N$-acetyl groups has not been experimentally proven yet. To test the function of the $N$-acetyl groups in NFs, we prepared derivatives from the $S$. meliloti $\operatorname{NodSm-IV}\left(\mathrm{C}_{16: 2}, \mathrm{~S}\right)$ that have glucosamine instead of $\mathrm{N}$-acetylglucosamine molecules in their carbohydrate core structure. These molecules were obtained by an in vitro $\mathrm{N}$-deacetylation reaction with a purified chitin deacetylase from the plant pathogen Colletotrichum lindemuthianum (Tokuyasu et al. 1996). Chitin deacetylases are enzymes that deacetylate the polymer chitin and its oligosaccharides (e.g., Trudel and Asselin 
1990; Kafetzopoulos et al. 1993; Alfonso et al. 1995; Tokuyasu et al. 1996, 1997). The N-deacetylated NF derivatives were purified, analyzed by mass spectrometry, and characterized with respect to their stability in the rhizosphere and their biological activity on the host plant $M$. sativa.

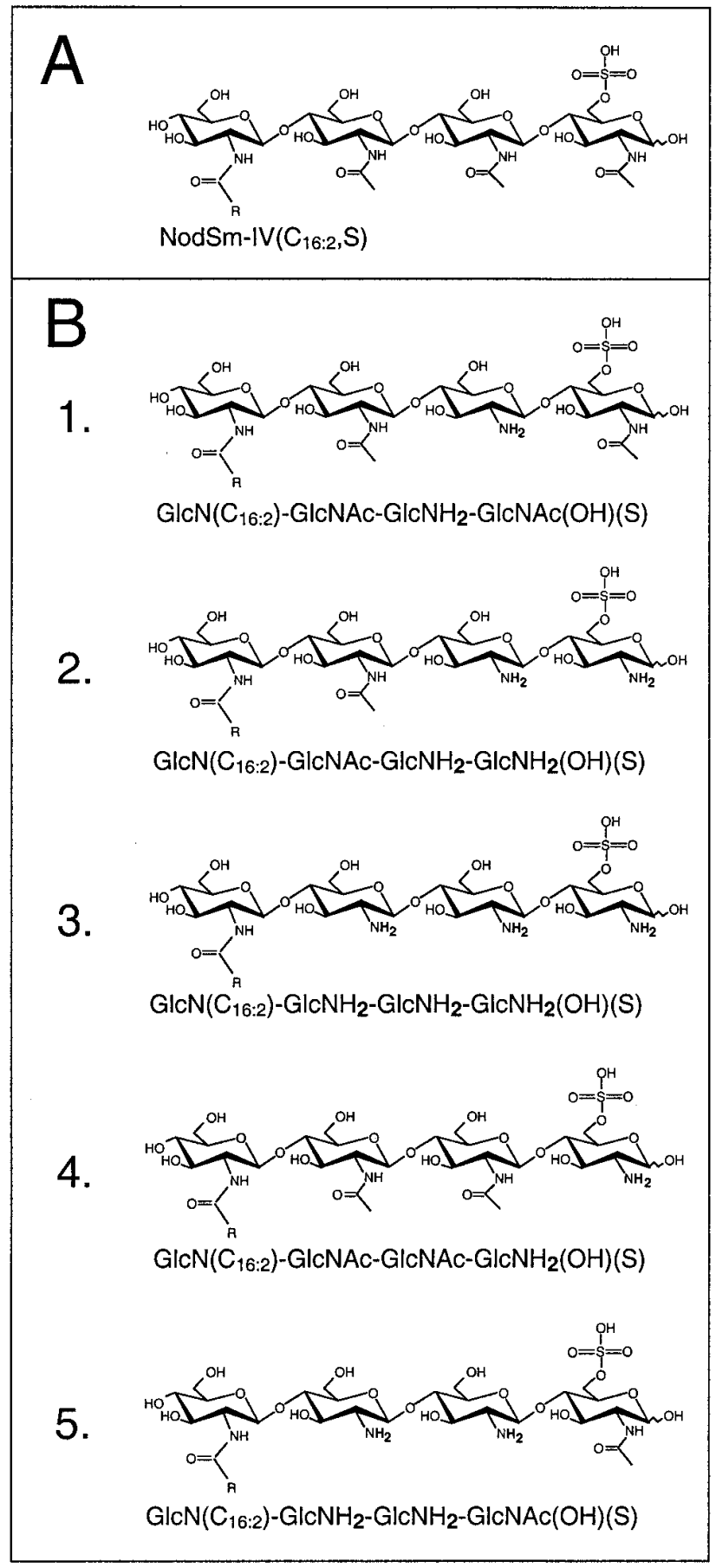

Fig. 1. Chemical structures. A, NodSm-IV $\left(\mathrm{C}_{16: 2}, \mathrm{~S}\right)$, the tetrameric and sulfated Nod factor from Sinorhizobium meliloti was used as substrate for the deacetylation reaction. B, Structure of the doubly $\mathrm{N}$-acetylated, mono-N-acetylated, and completely deacetylated derivatives identified by $\mathrm{MS} / \mathrm{MS}$. $\mathrm{R}=\mathrm{C}_{16: 2}$ acyl chain

\section{RESULTS}

\section{Deacetylation of NodSm-IV $\left(\mathrm{C}_{16: 2}, \mathrm{~S}\right)$ and purification of its $\mathrm{N}$-deacetylated derivatives.}

To test the requirement of the $N$-acetyl groups in NFs, we enzymatically prepared $\mathrm{N}$-deacetylated $\mathrm{NF}$ derivatives. $\operatorname{NodSm}-\mathrm{IV}\left(\mathrm{C}_{16: 2}, \mathrm{~S}\right)$, a tetrameric, sulfated NF purified from $S$. meliloti (Figs. 1A and 2A) was incubated with purified chitin deacetylase from the fungus $C$. lindemuthianum. This enzyme has the capacity to release $N$-acetyl groups from chitin and chitin oligomers as well (Tokuyasu et al. 1997). As shown in Figure 2, NodSm-IV $\left(\mathrm{C}_{16: 2}, \mathrm{~S}\right)$ was a substrate for the chitin deacetylase. After incubation, the reaction mixture was analyzed by $\mathrm{C}_{18}$ reverse-phase high-pressure liquid chromatography (HPLC). Incubation of NodSm-IV $\left(\mathrm{C}_{16: 2}, \mathrm{~S}\right)$ for $1 \mathrm{~h}$ resulted in several overlapping new peaks that showed a delayed retention time on the HPLC chromatograms (Fig. 2B). After 3 $\mathrm{h}$, solely $\mathrm{N}$-deacetylated derivatives were seen, while the substrate NodSm-IV $\left(\mathrm{C}_{16: 2}, \mathrm{~S}\right)$ was not detected anymore (Fig. $\left.2 \mathrm{C}\right)$.

Figure 3 summarizes the purification procedure that was used to separate the $\mathrm{N}$-deacetylated derivatives from traces of remaining, intact NodSm-IV $\left(\mathrm{C}_{16: 2}, \mathrm{~S}\right)$. After incubation of the NF with chitin deacetylase, the reaction mixture was acidified, loaded on a Dowex column, and finally eluted with $2 \% \mathrm{NH}_{3}$. A separate experiment confirmed that the intact NodSm$\operatorname{IV}\left(\mathrm{C}_{16: 2}, \mathrm{~S}\right)$ was not able to bind to the column under these conditions and that a mixture of $\operatorname{NodSm}-\mathrm{IV}\left(\mathrm{C}_{16: 2}, \mathrm{~S}\right)$ and its Ndeacetylated derivatives could be separated by this method (data not shown). After this purification step, the $\mathrm{N}$ deacetylated reaction products were purified by reverse-phase HPLC (35\% acetonitrile/water, $40 \mathrm{mM}$ ammonium acetate). Finally, the preparation was desalted with a Polygosil column and called purified "material 1."

Material 1 did not contain detectable amounts of intact $\operatorname{NodSm-IV}\left(\mathrm{C}_{16: 2}, \mathrm{~S}\right)$ as analyzed by HPLC chromatograms and mass spectrometry (data not shown). However, it was not excluded that the purified preparation still contained NodSm$\operatorname{IV}\left(\mathrm{C}_{16: 2}, \mathrm{~S}\right)$ in very low amounts. Therefore, an additional purification step to eliminate $\operatorname{NodSm-IV}\left(\mathrm{C}_{16: 2}, \mathrm{~S}\right)$ from the $\mathrm{N}$ deacetylated molecules was achieved with intact roots of the host plant $M$. sativa. We had shown previously that young $M$. sativa roots were able to rapidly hydrolyze NFs of $S$. meliloti (Staehelin et al. 1994b) and that $\left.\operatorname{NodSm-IV(C} C_{16: 2}, S\right)$ was degraded in the rhizosphere by an extracellular "lipodisaccharide forming" NF hydrolase (Staehelin et al. 1995; see also Figure 4). Moreover, we observed that active NFs were strongly bound to the root when nanomolar concentrations of NFs were added to the rhizosphere (C. Staehelin and M. Schultze, unpublished results). Hence, roots of the host plant $M$. sativa have a strong NF degradation activity and binding affinity for NodSm-IV $\left(\mathrm{C}_{16: 2}, \mathrm{~S}\right)$. Taking advantage of this, incubation of the $\mathrm{N}$-deacetylated NF derivatives (material 1) with intact $M$. sativa roots was applied as a method to eliminate the possible trace amounts of $\operatorname{NodSm}-\operatorname{IV}\left(\mathrm{C}_{16: 2}, \mathrm{~S}\right)$. When the N-deacetylated NF derivatives were incubated with intact roots for $24 \mathrm{~h}$, only small amounts of a hydrolytic degradation product were observed on HPLC chromatograms (Fig. 2E). This molecule, released from partially $N$-acetylated molecules, was identified by mass spectrometry as acetylated disaccharide $\operatorname{GlcN}\left(\mathrm{C}_{16: 2}\right)-\mathrm{GlcNAc}(\mathrm{OH})$ with a pseudomolecular ion at $m / z=617$ and a fragment ion of $\mathrm{m} / \mathrm{z}=396$ 
(data not shown). Most of the material, however, was not degraded by the $M$. sativa root. These nonhydrolyzed molecules present in the incubation medium were purified from the lipodisaccharide by reverse-phase HPLC, desalted, and called purified "material 2" (Figs. 2D and 3).

\section{Analysis by mass spectrometry.}

All fractions were analyzed by liquid secondary ion mass spectrometry (LSIMS). In the negative ionization mode, the presence of the deprotonated molecular ions $(\mathrm{M}-\mathrm{H})^{-}$of each species was expected. Both preparations (material 1 and material 2) exhibited mostly two peaks at $\mathrm{m} / \mathrm{z}, 1059.5$ and 1017.6 (Fig. 5). The first one corresponded to mono-N-deacetylated NodSm-IV $\left(\mathrm{C}_{16: 2}, \mathrm{~S}\right)$ (molecular weight 1060.5), and the second peak was due to doubly N-deacetylated $\operatorname{NodSm-IV}\left(\mathrm{C}_{16: 2}, \mathrm{~S}\right)$ (molecular weight 1018.6). In the positive ion mode, protonated molecules were generated, together with several fragment ions. The same molecules as above were identified with $\mathrm{MH}^{+}$ions at $\mathrm{m} / z 1061.4$ and 1019.4 , respectively, but a third one appeared with $\mathrm{MH}^{+}$at $m / z 977.4$ (Fig. 5). It was attributed to completely $\mathrm{N}$-deacetylated $\operatorname{Nod} \operatorname{Sm}-\mathrm{IV}\left(\mathrm{C}_{16: 2}, \mathrm{~S}\right.$ ) (molecular weight 976.4). All protonated molecules were accompanied by ions shifted down by $80 \mathrm{u}$ (loss of $\mathrm{SO}_{3}$ ) and by several fragments of the oligosaccharide backbone (Figs. 5 and 6). The molecular ion ratios of these different molecules were very different depending on the ionization mode. This was not surprising, as the different molecular species differed in their net electric charge. Thus, it was not possible to estimate their relative abundance from the mass spectra of the mixture.

To locate the position of the free amino groups in these partially N-deacetylated molecules, MS/MS (tandem mass spectrometry) spectra of the corresponding $\mathrm{MH}^{+}$ions were recorded. Main fragmentations occurred at the glycosidic bonds giving $\mathrm{B}$ ion series. The MS/MS spectrum of the $\mathrm{MH}^{+}$ion at $\mathrm{m} / \mathrm{z} 977.4$ (not shown) was consistent with the expected structure of completely N-deacetylated NodSm-IV $\left(\mathrm{C}_{16: 2}, \mathrm{~S}\right)$. Indeed, the main fragment intervals from the reducing end were $80 \mathrm{u}$ (loss of $\mathrm{SO}_{3}$ ), 179u (loss of glucosamine), two times $161 \mathrm{u}$ (loss of anhydroglucosamine). This series ended at $\mathrm{m} / \mathrm{z}$ 396 , which is the $\mathrm{B}_{1}$ ion at the nonreducing end bearing the $\mathrm{C}_{16: 2} N$-acyl substituent.

Removal of two $\mathrm{N}$-acetyl groups may give rise to three different products. The MS/MS spectrum of the corresponding $\mathrm{MH}^{+}$ion at $\mathrm{m} / \mathrm{z} 1019.4$ (Fig. 6) showed (from the reducing end) a $80 \mathrm{u}$ interval $\left(\mathrm{SO}_{3}\right)$, then either $179 \mathrm{u}\left(\mathrm{GlcNH}_{2} \mathrm{OH}\right)$ or 221u (GlcNAcOH), the former being prominent. The upper

Fig. 2. Analysis of NodSm-IV $\left(\mathrm{C}_{16: 2}, \mathrm{~S}\right)$ and its N-deacetylated derivatives by reverse-phase high-pressure liquid chromatography. A, NodSm$\operatorname{IV}\left(\mathrm{C}_{16: 2}, \mathrm{~S}\right)$, the Nod factor purified from Sinorhizobium meliloti. B, Formation of more slowly migrating, $\mathrm{N}$-deacetylated derivatives after incubation of NodSm-IV $\left(\mathrm{C}_{16: 2}, \mathrm{~S}\right)$ with chitin deacetylase for $1 \mathrm{~h}$. C, Same preparation incubated for $3 \mathrm{~h}$. D, Purified, N-deacetylated derivatives (material 2). E, Formation of the lipo-disaccharide $\mathrm{GlcN}\left(\mathrm{C}_{16: 2}\right)$ $\mathrm{GlcNAc}(\mathrm{OH})$ from N-deacetylated derivatives (material 1) after incubation with intact Medicago sativa roots for $24 \mathrm{~h}$. F, Purified Ndeacetylated derivatives (material 2) after incubation with intact $M$. sativa roots for $42 \mathrm{~h}$. Degradation products were not detectable. Peak 1: NodSm-IV $\left(\mathrm{C}_{16: 2}, \mathrm{~S}\right)$, purified from S. meliloti. Peak 2: N-deacetylated derivatives from NodSm-IV $\left(\mathrm{C}_{16: 2}, \mathrm{~S}\right)$. Peak 3: $\operatorname{GlcN}\left(\mathrm{C}_{16: 2}\right)-\operatorname{GlcNAc}(\mathrm{OH})$ separated into its anomers.

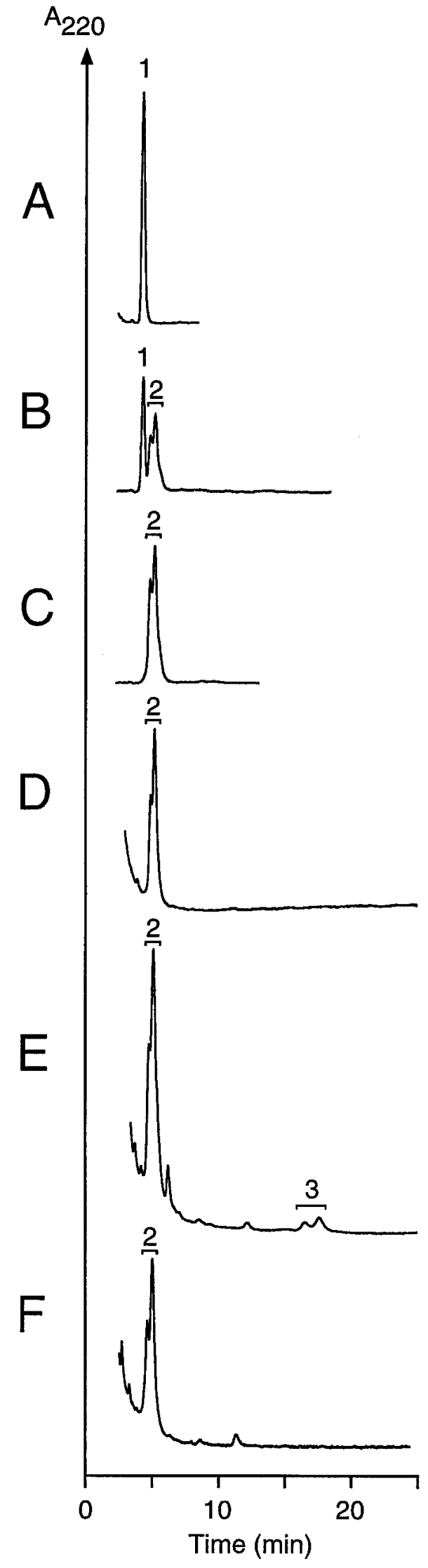


intervals from the $\mathrm{B}_{1}$ ion at $\mathrm{m} / \mathrm{z} 396$ were $203 \mathrm{u}$, mostly (anhydro GlcNAc) or $161 \mathrm{u}$ (anhydro $\mathrm{GlcNH}_{2}$ ). This interpretation indicated that the remaining $N$-acetyl group was partly on the reducing end and partly on the third residue from the reducing end, the most intense ions corresponding to the latter.

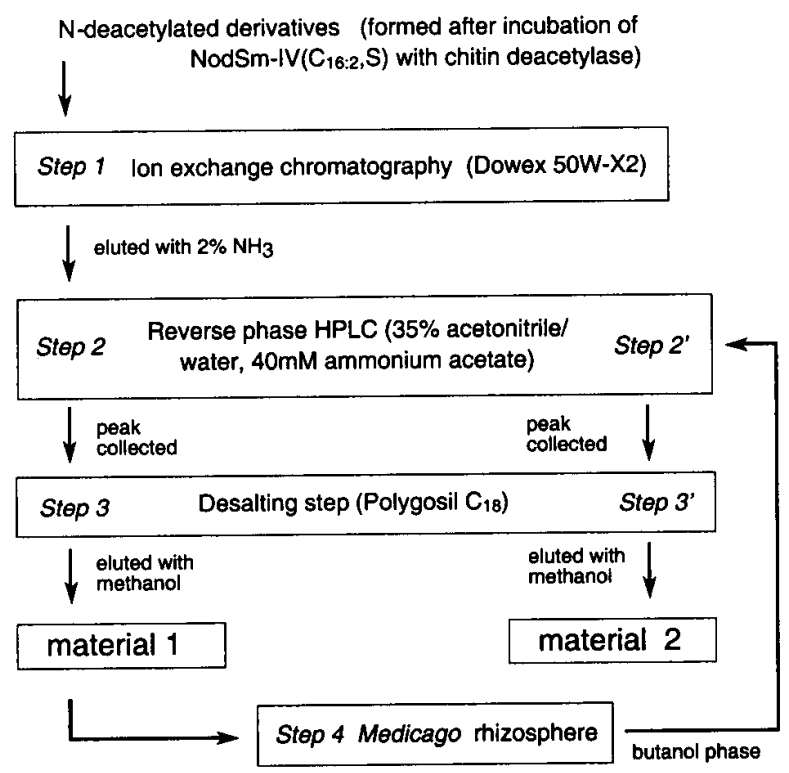

Fig. 3. Purification of N-deacetylated Nod factor derivatives called "material 1" and "material 2," respectively. Material 2 was obtained after incubation of material 1 with intact Medicago sativa roots, followed by a re-purification procedure.

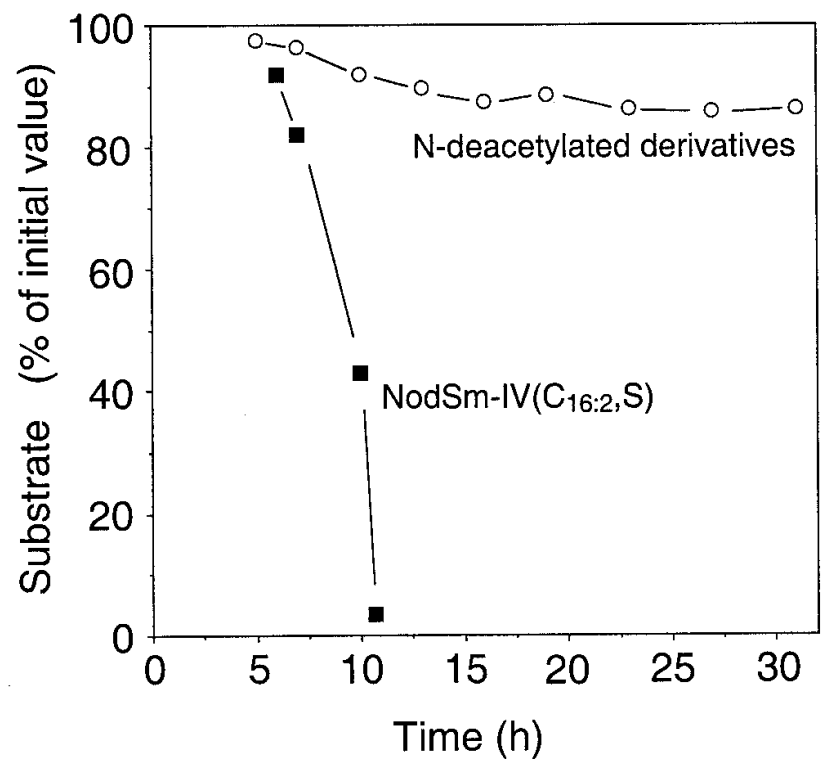

Fig. 4. Stability of NodSm-IV $\left(\mathrm{C}_{16: 2}, \mathrm{~S}\right)$ and its $\mathrm{N}$-deacetylated derivatives in the rhizosphere of Medicago sativa. $\operatorname{NodSm-IV}\left(\mathrm{C}_{16: 2}, \mathrm{~S}\right)$ or its $\mathrm{N}$ deacetylated derivatives (material 1) were incubated with intact $M$. sativa roots for different times. After incubation, samples were fractionated on reverse-phase high-pressure liquid chromatography and the ratio of substrate to the lipo-disaccharide $\mathrm{GlcN}\left(\mathrm{C}_{16: 2}\right)$ - $\mathrm{GlcNAc}(\mathrm{OH})$ product was determined. Each point represents the average value obtained from three seedlings.
From this spectrum, it could not be decided whether a $\mathrm{N}$ acetyl group was also present on the second residue or not.

The MS/MS spectrum from the doubly $N$-acetylated components $\left(\mathrm{MH}^{+}\right.$ion at $\left.m / z, 1061.4\right)$ exhibited, between the fragment $\mathrm{MH}-80$ and the subsequent ones, a $221 \mathrm{u}$ interval corresponding to $\mathrm{N}$-acetylglucosamine, thus locating this residue at the reducing end (Fig. 6). However, a weak ion with a $179 \mathrm{u}$ interval indicated that a less abundant component with glucosamine at the reducing end was also present. At the other side, a $203 \mathrm{u}$ interval was found between the $\mathbf{B}_{1}$ ion at $m / z, 396$ and the closer $\mathrm{B}_{2}$. A $161 \mathrm{u}$ interval, if any, corresponded to a signal with a very weak abundance. Thus, this glucosamine was mostly $\mathrm{N}$-acetylated.

Figure 1B shows the chemical structures identified by MS/MS analysis. The most abundant products were the mono$\mathrm{N}$-deacetylated GlcN $\left(\mathrm{C}_{16: 2}\right)$-GlcNAc-GlcNH${ }_{2}-\mathrm{GlcNAc}(\mathrm{OH})(\mathrm{S})$, the doubly N-deacetylated $\mathrm{GlcN}\left(\mathrm{C}_{16: 2}\right)$-GlcNAc-GlcNH${ }_{2}-$ $\mathrm{GlcNH}_{2} \mathrm{OH}(\mathrm{S})$, and the completely N-deacetylated GlcN $\left(\mathrm{C}_{16: 2}\right)$ $\mathrm{GlcNH}_{2}-\mathrm{GlcNH}_{2}-\mathrm{GlcNH}_{2}(\mathrm{OH})(\mathrm{S})$. Furthermore, two minor components were found: The mono-N-deacetylated $\mathrm{GlcN}\left(\mathrm{C}_{16: 2}\right)$ GlcNAc-GlcNAc-GlcNH ${ }_{2}(\mathrm{OH})(\mathrm{S})$ and the doubly N-deacetylated $\mathrm{GlcN}\left(\mathrm{C}_{16: 2}\right)-\mathrm{GlcNH}_{2}-\mathrm{GlcNH}_{2}-\mathrm{GlcNAc}(\mathrm{OH})$ (S). Since the polarity and the hydrophobicity of the $\mathrm{N}$-deacetylated molecules are very different, it was not possible to exactly determine their relative abundance from the MS/MS spectra.

\section{High stability of $\mathrm{N}$-deacetylated NF derivatives in the $M$. sativa rhizosphere.}

When incubated with intact $M$. sativa roots, NodSm$\operatorname{IV}\left(\mathrm{C}_{16: 2}, \mathrm{~S}\right)$ was rapidly hydrolyzed and inactivated by a specific "lipo-disaccharide forming" NF hydrolase. It was hypothesized that inactivation of NFs after their perception is an important step of symbiotic signaling (Staehelin et al. 1994b, 1995). Therefore, we tested the stability of N-deacetylated derivatives in the $M$. sativa rhizosphere, compared with NodSm-IV $\left(\mathrm{C}_{16: 2}, \mathrm{~S}\right)$. A time course experiment shown in Figure 4 illustrates the difference in stability of N-deacetylated derivatives (material 1) compared with intact NodSm-IV $\left(\mathrm{C}_{16: 2}, \mathrm{~S}\right)$ in the rhizosphere of $M$. sativa. Roots of young seedlings were incubated in a solution containing NodSm-IV $\left(\mathrm{C}_{16: 2}, \mathrm{~S}\right)$ or its derivatives for different time periods. After extraction of the medium with $n$-butanol, the substrate and the

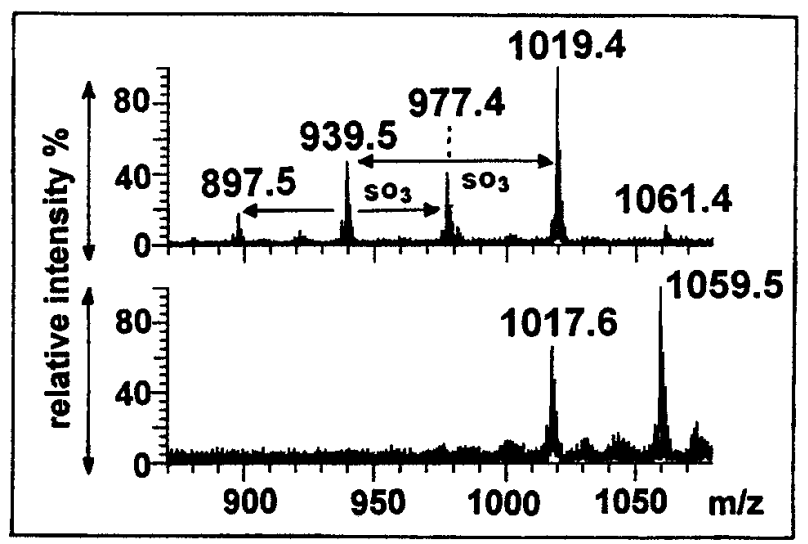

Fig. 5. Liquid secondary ion mass spectrometry (LSIMS) spectra of the partially N-deacetylated Nod factor derivatives. Molecular region of the positive (top) and negative ion ( bottom) LSIMS spectra. 
acylated cleavage products were fractionated by reverse-phase HPLC. The graph indicates the percentage of nonhydrolyzed substrate after a given incubation time. The NodSm-IV $\left(\mathrm{C}_{16: 2}, \mathrm{~S}\right)$ molecules were rapidly degraded to the lipo-disaccharide $\operatorname{GlcN}\left(\mathrm{C}_{16: 2}\right)-\mathrm{GlcNAc}(\mathrm{OH})$, likely by the "lipodisaccharide forming" NF hydrolase as reported previously (Staehelin et al. 1995). In contrast, only small amounts of $\mathrm{GlcN}\left(\mathrm{C}_{16: 2}\right)$-GlcNAc(OH) were released from the deacetylated derivatives (material 1) (Fig. 2E). After about $16 \mathrm{~h}$, a plateau was reached and no further hydrolysis was observed (Fig. 4). These findings were confirmed by an additional experiment. After re-purification of the nonhydrolyzed molecules, the material (i.e., material 2) was incubated for a second time with intact roots of freshly prepared $M$. sativa plants. As expected, these molecules were resistant against hydrolysis, even after an incubation time as long as $42 \mathrm{~h}$ (Fig. 2F). Similar results were obtained when a mixture of NodSm$\operatorname{IV}\left(\mathrm{C}_{16: 2}, \mathrm{~S}\right)$ and its $\mathrm{N}$-deacetylated derivatives was tested. NodSm-IV $\left(\mathrm{C}_{16: 2}, \mathrm{~S}\right)$ was degraded to the lipo-disaccharide, while the deacetylated derivatives remained intact (not shown). These data indicate that the deacetylated derivatives exhibit a high stability in the $M$. sativa rhizosphere and that the $N$-acetyl groups of NFs make the carbohydrate moiety accessible for hydrolases of the host plant.
Low biological activity of $\mathrm{N}$-deacetylated NF derivatives.

NFs are known to induce a number of specific responses on the host plants. To test whether the deacetylated derivatives show a similar inducing activity on $M$. sativa, two different bioassays were used, i.e., the capacity to stimulate root hair deformation and to enhance "lipo-disaccharide forming" NF hydrolase activity in the rhizosphere (Staehelin et al. 1995).

In a first series of experiments, the activity to stimulate root hair deformation on young $M$. sativa roots was determined. As seen in Table 1 , the intact NF was able to induce a response with a threshold at $10^{-11} \mathrm{M}$. When deacetylated derivatives (material 2) were tested, a threshold at about $10^{-7} \mathrm{M}$ was detected, indicating an approximately 10,000-fold lower activity, compared with NodSm-IV $\left(\mathrm{C}_{16: 2}, \mathrm{~S}\right)$. Excess amounts of deacetylated derivatives were not able to inhibit the root hair deformation responses induced by intact $\operatorname{NodSm-IV}\left(\mathrm{C}_{16: 2}, \mathrm{~S}\right)$, indicating that the deacetylated molecules cannot act as competitive inhibitors (not shown).

In a second series of experiments, the deacetylated derivatives were tested for their ability to stimulate the "lipodisaccharide forming" NF hydrolase of $M$. sativa (Staehelin et al. 1995). The activity of this enzyme showed a concentrationdependent stimulation when $M$. sativa roots were pretreated with active NFs. For NodSm-IV $\left(\mathrm{C}_{16: 2}, \mathrm{~S}\right)$, a threshold concen-

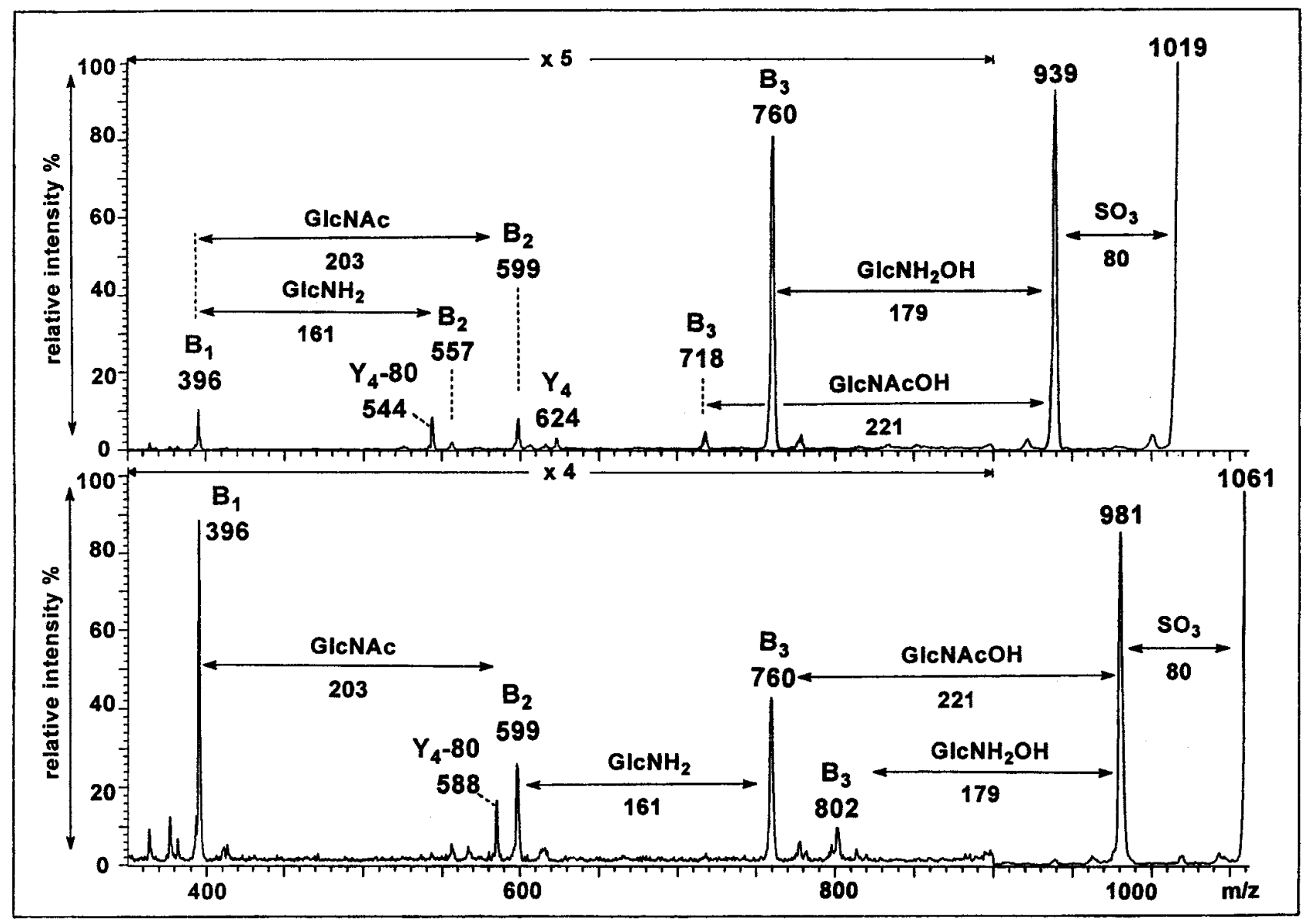

Fig. 6. MS/MS spectra of the partially N-deacetylated Nod factor derivatives. MS/MS spectra of the $\mathrm{MH}^{+}$ions from doubly (top) and mono (bottom) Ndeacetylated species at $\mathrm{m} / z 1019$ and 1061, respectively. The B fragments resulted from glycosidic cleavages with charge retention on the nonreducing part. The Y fragments are their counterpart with charge retention on the reducing end. The mass range was scanned from $\mathrm{MH}^{+}$down to $m / z 350$. 
tration of $10^{-8} \mathrm{M}$ was measured (Staehelin et al. 1995). As seen in Figure 7, a pretreatment of roots with $10^{-6} \mathrm{M}$ NodSm$\operatorname{IV}\left(\mathrm{C}_{16: 2}, \mathrm{~S}\right)$ resulted in a fourfold stimulation of the enzyme activity (column 3 ). The $\mathrm{N}$-deacetylated derivatives (material 2) were not able to stimulate the hydrolase (column 2), i.e., the "lipo-disaccharide forming" activity of pretreated plants was as low as for the control plants, which were mock-treated with Jensen medium (column 1). Addition of excess amounts of $\mathrm{N}$-deacetylated derivatives (material 2) did not influence the effect induced by active NFs (column 4). These results confirm and extend the data obtained from the root hair deformation assay and show that N-deacetylation of NFs dramatically decreases their biological activity.

\section{DISCUSSION}

In this paper, we report the preparation of $\mathrm{N}$-deacetylated derivatives of NodSm-IV $\left(\mathrm{C}_{16: 2}, \mathrm{~S}\right)$ with chitin deacetylase from $C$. lindemuthianum. The enzyme was able to release all three $\mathrm{N}$-acetyl groups of the tetrameric NF and produced a family of doubly $\mathrm{N}$-acetylated, mono-N-acetylated, and completely deacetylated molecules. The rhizobial NodB protein involved in NF synthesis, however, specifically $\mathrm{N}$-deacetylates a single acetyl group at the nonreducing end of chitin oligomers, forming mono-deacetylated precursor molecules that can be acylated by NodA protein (John et al. 1993). Despite this difference in substrate specificity, fungal genes encoding chitin deacetylase show remarkable sequence similarities with $\operatorname{nodB}$ (Kafetzopoulos et al. 1993).

Compared with intact $\operatorname{NodSm-IV}\left(\mathrm{C}_{16: 2}, \mathrm{~S}\right)$, the N-deacetylated derivatives displayed an approximately 10,000-fold reduced biological activity in the root hair deformation assay. After incubation with chitin deacetylase, intact NFs were not detected anymore on HPLC chromatograms (Fig. 2C). The following four purification steps further removed trace amounts of NFs from the N-deacetylated derivatives. We assume that each of these steps resulted in an approximately 100-fold reduction of contaminating $\operatorname{NodSm-IV}\left(\mathrm{C}_{16: 2}, \mathrm{~S}\right)$. However, we cannot completely exclude that the weak biological activity of the $\mathrm{N}$-deacetylated derivatives in inducing root hair deformation was due to contaminating NodSm$\operatorname{IV}\left(\mathrm{C}_{16: 2}, \mathrm{~S}\right)$. Hence, it is possible that $\mathrm{N}$-deacetylated derivatives are completely inactive in eliciting root hair deformation on $M$. sativa roots. It is worth noting in this context, however, that also the lipo-disaccharide $\operatorname{GlcN}\left(\mathrm{C}_{16: 2}\right)-\operatorname{GlcNAc}(\mathrm{OH})$ or the lipo-trisaccharide $\operatorname{GlcN}\left(\mathrm{C}_{16: 2}\right)$-GlcNAc-GlcNAc(OH) retained root hair deformation activity in the micromolar concentration range (Staehelin et al. 1994b).

Table 1. Root hair deformation assay ${ }^{\mathrm{a}}$

\begin{tabular}{|c|c|c|c|c|c|c|c|}
\hline \multirow[b]{2}{*}{ Treatment } & \multicolumn{7}{|c|}{ Concentration (M) } \\
\hline & $10^{-5}$ & $10^{-6}$ & $10^{-7}$ & $10^{-8}$ & $10^{-9}$ & $10^{-10}$ & $10^{-11}$ \\
\hline $\operatorname{NodSm-IV}\left(\mathrm{C}_{16: 2}, \mathrm{~S}\right)$ & + & + & + & + & + & + & \pm \\
\hline $\begin{array}{l}\text { N-deacetylated de- } \\
\text { rivatives }\end{array}$ & + & + & \pm & - & - & - & - \\
\hline \multicolumn{8}{|c|}{$\begin{array}{l}\text { Capacity of NodSm-IV }\left(\mathrm{C}_{16: 2}, \mathrm{~S}\right) \text { and its } \mathrm{N} \text {-deacetylated derivatives } \\
\text { (material 2) to induce root hair deformation on Medicago sativa was } \\
\text { tested at indicated concentrations. Positive and negative scores were } \\
\text { given when root hair deformation was present }(+) \text { or absent }(-) \text {, re- } \\
\text { spectively. At the threshold value }( \pm) \text { only a few plants were respon- } \\
\text { sive. }\end{array}$} \\
\hline
\end{tabular}

A treatment of roots with excess amounts of N-deacetylated derivatives did not inhibit the responses of the host plant induced by intact NFs. We suggest that N-deacetylated derivatives do not bind to the NF receptors involved in root hair deformation and stimulation of the "lipo-disaccharide forming" NF hydrolase. Hence, these receptors seem to have a strongly reduced affinity for lipo-oligosaccharides when GlcNAc of the core structure is replaced by $\mathrm{GlcNH}_{2}$.

We have found previously that tetrameric NFs of $S$. meliloti are rapidly degraded by host plants but are highly stable in the rhizosphere of nonhost plants (Staehelin et al. 1994b, 1997). It is tempting to speculate that NF degradation is a prerequisite for a compatible symbiotic interaction and that host plants have evolved specific enzymes that cleave and inactivate the rhizobial signal molecules (Staehelin et al. 1995). In the present study, the release of $N$-acetyl groups from NFs resulted in a dramatically increased stability in the $M$. sativa rhizosphere. These results indicate that the $\mathrm{N}$-deacetylated derivatives (material 2) are substrates for neither the "lipo-disaccharide forming" NF hydrolase nor other glycosyl hydrolases in the $M$. sativa rhizosphere.

It has been reported by Bec-Ferté et al. (1996) that certain $S$. fredii strains produce various NFs including a compound with glucose inserted to the chitin oligomer chain. This pentameric NF was found to be degradable by a bacterial chitinase; however, its biological activity on roots was not examined. Based on our results with N-deacetylated NF derivatives, we suggest that this glucose-containing NF has low biological activity on host plants.

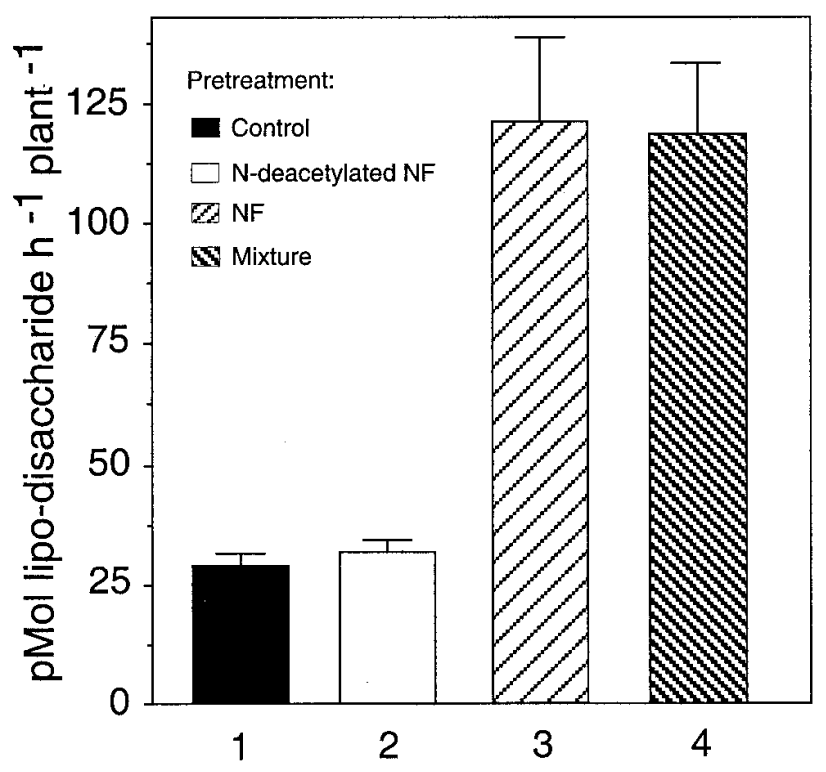

Fig. 7. Capacity of NodSm-IV $\left(\mathrm{C}_{16: 2}, \mathrm{~S}\right)$ and its N-deacetylated derivatives to stimulate the "lipo-disaccharide forming" Nod factor (NF) hydrolase of Medicago sativa. Roots of young M. sativa seedlings were pretreated for $19 \mathrm{~h}$ with the following solutions: Jensen medium as a control (column 1); $1 \mu \mathrm{M}$ N-deacetylated derivatives (material 2) (column 2); $1 \mu \mathrm{M}$ NodSm-IV $\left(\mathrm{C}_{16: 2}, \mathrm{~S}\right)$ (column 3); and a mixture of 10 $\mu \mathrm{M}$ N-deacetylated derivatives with $1 \mu \mathrm{M} \operatorname{NodSm-IV}\left(\mathrm{C}_{16: 2}, \mathrm{~S}\right)$ (column 4). After incubation, roots were incubated in $5 \mu \mathrm{M} \operatorname{NodSm}-\operatorname{IV}\left(\mathrm{C}_{16: 2}, \mathrm{~S}\right)$ for $2 \mathrm{~h}$. Release of the lipo-disaccharide $\operatorname{GlcN}\left(\mathrm{C}_{16: 2}\right)-\operatorname{GlcNAc}(\mathrm{OH})$ from the NF substrate was analyzed by reverse-phase high-pressure liquid chromatography. Standard deviation of the mean $(n=3)$ is indicated. 
In addition to $\operatorname{NodSm-IV}\left(\mathrm{C}_{16: 2}, \mathrm{~S}\right)$, we were able to $\mathrm{N}$ deacetylate various other $\mathrm{NFs}$, including the $\mathrm{O}$-acetylated NodSm-IV $\left(\mathrm{C}_{16: 2}, \mathrm{Ac}, \mathrm{S}\right)$ and the nonsulfated derivative NodSm$\operatorname{IV}\left(\mathrm{C}_{16: 2}\right)$ (data not shown). It is possible that all rhizobial NFs are substrates for the chitin deacetylase from $C$. lindemuthianum. The production of $\mathrm{N}$-deacetylated derivatives opens the possibility to reacetylate them chemically by radioactively labeled acetic anhydride, as described for chitosan that can be converted to chitin (e.g., Molano et al. 1977; Boller 1992). Radioactively labeled NFs are valuable tools to characterize NF-binding proteins, which are candidates for putative NF receptors. For in vitro radiolabeling, NFs were chemically modified, e.g., by catalytic hydrogenation, forming less active NFs (Schultze et al. 1992). Alternatively, specific enzymes were required, such as the sulfate transferase NodH (Schultze et al. 1995; Bourdineaud et al. 1995) and the $O$-acetyl transferase NodL (Bloemberg et al. 1994). Enzymatic Ndeacetylation and reacetylation with acetic anhydride, however, would be a general method for in vitro radiolabeling of any NF, without modifying its structure.

\section{MATERIALS AND METHODS}

\section{Nod factors and chitin deacetylase.}

Purification of the tetrameric and sulfated NodSm-IV $\left(\mathrm{C}_{16: 2}, \mathrm{~S}\right)$ produced by the $S$. meliloti (formerly named Rhizobium meliloti) strain 1021(pEK327) has been described previously (Schultze et al. 1992). Chitin deacetylase from a culture filtrate of the fungus $C$. lindemuthianum (ATCC56676) was purified to electrophoretic homogeneity as reported (Tokuyasu et al. 1996), lyophilized in aliquots, and resuspended in $\mathrm{H}_{2} \mathrm{O}$ for the enzymatic reaction with NFs.

\section{Deacetylation reaction.}

For a typical in vitro deacetylation reaction, $1 \mathrm{mg}$ of $\operatorname{NodSm-IV}\left(\mathrm{C}_{16: 2}, \mathrm{~S}\right)$ was incubated with $2 \mathrm{U}$ of purified chitin deacetylase in $0.5 \mathrm{ml}$ of $20 \mathrm{mM}$ sodium phosphate buffer, $\mathrm{pH}$ 7.5 , at $37^{\circ} \mathrm{C}$ for $3 \mathrm{~h}$. The deacetylation reaction was monitored by fractionating small aliquots on reverse-phase HPLC under isocratic conditions with $35 \%$ acetronitrile/water, $40 \mathrm{mM}$ ammonium acetate as the mobile phase (Staehelin et al. $1994 b)$. NodSm-IV $\left(\mathrm{C}_{16: 2}, \mathrm{~S}\right)$ and the more slowly migrating $\mathrm{N}$ deacetylated derivatives were detected by their absorption at $220 \mathrm{~nm}$. Quantification of the N-deacetylated derivatives was based on their absorption of the $\mathrm{C}_{16: 2}$ acyl chain at $220 \mathrm{~nm}$.

\section{Purification of $\mathbf{N}$-deacetylated derivatives (material 1).}

Step 1: Ion exchange chromatography (according to Drouillard et al. 1997). After incubation, the sample was acidified with $50 \mu \mathrm{l}$ of $1 \mathrm{M}$ acetic acid and loaded onto a Dowex 50W-X2 column (150 mg dry weight), which was equilibrated with $10 \mathrm{mM}$ acetic acid. As a control, $\operatorname{NodSm-IV}\left(\mathrm{C}_{16: 2}, \mathrm{~S}\right)$ was chromatographed under the same conditions. After the columns were washed with $\mathrm{H}_{2} \mathrm{O}$, aliquots were analyzed by reverse-phase HPLC (see above). While $\operatorname{NodSm-IV}\left(\mathrm{C}_{16: 2}, \mathrm{~S}\right)$ was found in the flow-through, the charged $\mathrm{N}$-deacetylated derivatives bound to the column and could be eluted with $3 \mathrm{ml}$ of $2 \%$ ( $\mathrm{vol} / \mathrm{vol}$ ) aqueous $\mathrm{NH}_{3}$.

Step 2: Reverse-phase HPLC. The eluted, N-deacetylated derivatives were concentrated under reduced pressure and the residue (a few microliters) was taken up in $100 \mu \mathrm{l}$ of $35 \%$ acetronitrile/water, $40 \mathrm{mM}$ ammonium acetate. The sample was purified by reverse-phase HPLC as described (Staehelin et al. 1994b) with $35 \%$ acetronitrile/water, $40 \mathrm{mM}$ ammonium acetate as the mobile phase. The peak representing the $\mathrm{N}$ deacetylated compounds was collected and 20-fold concentrated under reduced pressure.

Step 3: Desalting step. A $\mathrm{C}_{18}$ column (Machery Nagel, Düren, Germany, Polygosil $\mathrm{C}_{18}, 60-4063$, particle size 40 to $63 \mu \mathrm{m}$ ) was washed with $100 \%$ methanol and equilibrated with $\mathrm{H}_{2} \mathrm{O}$. The $\mathrm{N}$-deacetylated compounds were diluted in 1 $\mathrm{ml}$ of $\mathrm{H}_{2} \mathrm{O}$ and loaded onto the column (200 mg dry weight). The column was washed with $10 \mathrm{ml}$ of $\mathrm{H}_{2} \mathrm{O}$ and the deacetylated derivatives were then eluted with $1 \mathrm{ml}$ of $100 \%$ methanol and dried in a Speed-Vac evaporator.

\section{Purification of $\mathbf{N}$-deacetylated derivatives (material 2).}

Step 4: Incubation in the $M$. sativa rhizosphere. Ndeacetylated derivatives (material 1) were incubated with germinated $M$. sativa (cv. Sitel) seedlings in 1-ml sterile plastic syringes as described previously (Staehelin et al. 1994b). Briefly, $100 \mu \mathrm{g}$ of material 1 was taken up in $10 \mathrm{ml}$ of depositfree Jensen medium containing 0.5 ( $\mathrm{vol} / \mathrm{vol}$ ) dimethyl sulfoxide (DMSO). The solution was filled into the plastic syringes (200 $\mu$ l per syringe) and then a young seedling was placed on top of each syringe. After incubation at $24^{\circ} \mathrm{C}$ in the dark for $24 \mathrm{~h}$, the seedlings were removed and the contents of the syringes were pooled and extracted with $3 \mathrm{ml}$ of distilled $n$ butanol. The butanol phase was dried in a Speed-Vac evaporator.

Step 2': Reverse-phase HPLC as described for step 2.

Step 3': Samples were desalted as described for step 3.

\section{Analysis by mass spectrometry.}

All spectra were recorded on an AutoSpec instrument (Micromass, Manchester, UK) fitted with an LSIMS source and a cesium ion gun. The accelerating voltage was set to 8 $\mathrm{kV}$, the cesium gun to $30 \mathrm{kV}$. Typically, samples (1 $\mu \mathrm{g}$ or less) were deposited on the LSIMS tip from a water solution and mixed with the appropriate matrix (1:1 glycerol/metanitrobenzyl alcohol, either acidified with $10 \%$ trichloroacetic acid for the positive ion mode or alkalinized with diisopropylmethyl amine for negative ions). MS/MS spectra were recorded in the constant B/E scanning mode.

\section{Hydrolysis of NodSm-IV $\left(\mathrm{C}_{16: 2}, \mathrm{~S}\right)$ and}

its $\mathrm{N}$-deacetylated derivatives by $M$. sativa roots.

The in vivo assay with intact $M$. sativa roots was performed as described previously (Staehelin et al. 1994b, 1995). Briefly, roots of $M$. sativa seedlings were incubated in 1-ml plastic syringes filled with $200 \mu \mathrm{l}$ of Jensen medium containing $0.5 \%$ (vol/vol) DMSO and $10 \mu \mathrm{M} \operatorname{NodSm-IV}\left(\mathrm{C}_{16: 2}, \mathrm{~S}\right)$ or its $\mathrm{N}$ deacetylated derivatives, respectively. After incubation at $24^{\circ} \mathrm{C}$ in the dark, the seedlings were removed. The culture medium of three seedlings was pooled and extracted with an equal volume of $n$-butanol. Substrates and the acylated degradation product $\operatorname{GlcN}\left(\mathrm{C}_{16: 2}\right)-\mathrm{GlcNAc}(\mathrm{OH})$ were fractionated on reverse-phase HPLC with $35 \%$ acetonitrile/water, $40 \mathrm{mM}$ ammonium acetate.

\section{Bioassays.}

As a bioassay for NF activity, two specific host plant responses were examined, root hair deformation and stimulation 
of the "lipo-disaccharide forming" NF hydrolase of $M$. sativa. The root hair deformation assay was performed as reported previously with 20 seedlings for each concentration range (Staehelin et al. 1994b). The activity of the "lipo-disaccharide forming" NF hydrolase was measured as described (Staehelin et al. 1995). Briefly, M. sativa seedlings were incubated for 19 $\mathrm{h}$ in syringes filled with a given concentration of NFs or Ndeacetylated derivatives, respectively. Plants were then transferred to new syringes containing $5 \mu \mathrm{M} \operatorname{NodSm}-\operatorname{IV}\left(\mathrm{C}_{16: 2}, \mathrm{~S}\right)$ and incubated at $24^{\circ} \mathrm{C}$ in the dark for $2 \mathrm{~h}$. After extraction with $n$-butanol, the substrate and the formed lipo-disaccharide $\operatorname{GlcN}\left(\mathrm{C}_{16: 2}\right)$-GlcNAc(OH) were analyzed by reverse-phase HPLC as described (Staehelin et al. 1994b).

\section{ACKNOWLEDGMENTS}

This work was partially supported by the Max Planck Research Award to A. K. We appreciated helpful discussions with Peter Mergaert (ISV, CNRS, Gif-sur-Yvette, France).

\section{LITERATURE CITED}

Alfonso, C., Nuero, O. M., Santamaria, F., and Reyes, F. 1995. Purification of a heat-stable chitin deacetylase from Aspergillus nidulans and its role in cell wall degradation. Curr. Microbiol. 30:49-54.

Bec-Ferté, M.-P., Krishnan, H. B., Savagnac, A., Pueppke, S. G., and Promé, J.-C. 1996. Rhizobium fredii synthesizes an array of lipooligosaccharides, including a novel compound with glucose inserted into the backbone of the molecule. FEBS Lett. 393:273-279.

Bladergroen, M. R., and Spaink, H. P. 1998. Genes and signal molecules involved in the rhizobia-Leguminoseae symbiosis. Curr. Opin. Plant Biol. 1:353-359.

Bloemberg, G. V., Thomas-Oates, J. E., Lugtenberg, B. J. J., and Spaink, H. P. 1994. Nodulation protein NodL of Rhizobium leguminosarum Oacetylates lipo-oligosaccharides, chitin fragments and $\mathrm{N}$-acetylglucosamine in vitro. Mol. Microbiol. 11:793-804.

Boller, T. 1992. Biochemical analysis of chitinase and $\beta$-1,3-glucanases. Pages 23-30 in: Practical Methods in Molecular Plant Pathology. D. J. Bowles and S. Gurr, eds. Oxford University Press, Oxford.

Bourdineaud, J. P., Bono, J. J., Ranjeva, R., and Cullimore, J. V. 1995. Enzymatic radiolabelling to a high specific activity of legume lipooligosaccharidic nodulation factors from Rhizobium meliloti. Biochem. J. 306:259-264.

Cohn, J., Day, R. B., and Stacey, G. 1998. Legume nodule organogenesis. Trends Plant Sci. 3:105-110.

Drouillard, S., Armand, S., Davies, G. J., Vorgias, C. E., and Henrissat, B. 1997. Serratia marcescens chitobiase is a retaining glycosidase utilizing substrate acetamido group participation. Biochem. J. 328: 945-949.

Geremia, R. A., Mergaert, P., Geelen, D., Van Montagu, M., and Holsters, M. 1994. The NodC protein of Azorhizobium caulinodans is an $\mathrm{N}$-acetylglucosaminyltransferase. Proc. Natl. Acad. Sci. USA 91: 2669-2673.

John, M., Röhrig, H., Schmidt, J., Wieneke, U., and Schell, J. 1993. Rhizobium NodB protein involved in nodulation signal synthesis is a chitooligosaccharide deacetylase. Proc. Natl. Acad. Sci. USA 90:625629.

Kafetzopoulos, D., Thireos, G., Vournakis, J. N., and Bouriotis, V. 1993. The primary structure of a fungal chitin deacetylase reveals the function for two bacterial gene products. Proc. Natl. Acad. Sci. USA 90: 8005-8008.
Kamst, E., Bakkers, J., Quaedvlieg, N. E. M., Pilling, J., Kijne, J. W., Lugtenberg, B. J. J., and Spaink, H. P. 1999. Chitin oligosaccharide synthesis by rhizobia and zebrafish embryos starts by glycosyl transfer to $\mathrm{O} 4$ of the reducing-terminal residue. Biochemistry 38:40454052 .

Long, S. R. 1996. Rhizobium symbiosis: Nod factors in perspective. Plant Cell 8:1885-1898.

Minic, Z., Brown, S., De Kouchkovsky, Y., Schultze, M., and Staehelin, C. 1998. Purification and characterization of a novel chitinaselysozyme, of another chitinase, both hydrolysing Rhizobium meliloti Nod factors, and of a pathogenesis-related protein from Medicago sativa roots. Biochem. J. 332:329-335.

Molano, J., Duran, A., and Cabib, E. 1977. A rapid and sensitive assay for chitinase using tritiated chitin. Anal. Biochem. 83:648-656.

Promé, J.-C. 1996. Signalling events elicited in plants by defined oligosaccharide structures. Curr. Opin. Struct. Biol. 6:671-678.

Roche, P., Debellé, F., Maillet, F., Lerouge, P., Faucher, C., Truchet, G., Dénarie, J., and Promé, J.-C. 1991. Molecular basis of symbiotic host specificity in Rhizobium meliloti: nodH and nodPQ genes encode the sulfation of lipo-oligosaccharide signals. Cell 67:1131-1143.

Schultze, M., and Kondorosi, A. 1998. Regulation of symbiotic root nodule development. Annu. Rev. Genet. 32:33-57.

Schultze, M., Quiclet-Sire, B., Kondorosi, E., Virelizier, H., Glushka, J. N., Endre, G., Géro, S. D., and Kondorosi, A. 1992. Rhizobium meliloti produces a family of sulfated lipooligosaccharides exhibiting different degrees of plant host specificity. Proc. Natl. Acad. Sci. USA 89:192-196.

Schultze, M., Staehelin, C., Brunner, F., Genetet, I., Legrand, M., Fritig, B., Kondorosi, E., and Kondorosi, A. 1998. Plant chitinase/lysozyme isoforms show distinct substrate specificity and cleavage site preference towards lipochitooligosaccharide Nod signals. Plant J. 16:571580 .

Schultze, M., Staehelin, C., Röhrig, H., John, M., Schmidt, J., Kondorosi, E., Schell, J., and Kondorosi, A. 1995. In vitro sulfotransferase activity of Rhizobium meliloti NodH protein: Lipochitooligosaccharide nodulation signals are sulfated after synthesis of the core structure. Proc. Natl. Acad. Sci. USA 92:2706-2709.

Staehelin, C., Granado, J., Müller, J., Wiemken, A., Mellor, R. B., Felix, G., Regenass, M., Broughton, W. J., and Boller, T. 1994a. Perception of Rhizobium nodulation factors by tomato cells and inactivation by root chitinases. Proc. Natl. Acad. Sci. USA 91:2196-2200.

Staehelin, C., Schultze, M., Kondorosi, E., and Kondorosi, A. 1995. Lipo-chitooligosaccharide nodulation signals from Rhizobium meliloti induce their rapid degradation by the host plant alfalfa. Plant Physiol. 108:1607-1614.

Staehelin, C., Schultze, M., Kondorosi, E., Mellor, R. B., Boller, T., and Kondorosi, A. 1994b. Structural modifications in Rhizobium meliloti Nod factors influence their stability against hydrolysis by root chitinases. Plant J. 5:319-330.

Staehelin, C., Vanney, M., Foucher, F., Kondorosi, E., Schultze, M., and Kondorosi, A. 1997. Degradation of nodulation signals from Rhizobium meliloti by its host plants. Pages 43-46 in: Biological Fixation of Nitrogen for Ecology and Sustainable Agriculture. A. Legocki, H. Bothe, and A. Pühler, eds. Springer, Berlin.

Tokuyasu, K., Ohnishi-Kameyama, M., and Hayashi, K. 1996. Purification and characterization of extracellular chitin deacetylase from Colletotrichum lindemuthianum. Biosci. Biotechnol. Biochem. 60: 1598-1603.

Tokuyasu, K., Ono, H., Ohnishi-Kameyama, M., Hayashi, K., and Mori, Y. 1997. Deacetylation of chitin oligosaccharides of dp 2-4 by chitin deacetylase from Colletotrichum lindemuthianum. Carbohydr. Res. 303:353-358

Trudel, J., and Asselin, A. 1990. Detection of chitin deacetylase activity after polyacrylamide gel electrophoresis. Anal. Biochem. 189:249253. 